\title{
NÍVEL DE ATIVIDADE FÍSICA EM PESSOAS COM SÍNDROME DE DOWN: UMA REVISÃO SISTEMÁTICA
}

\author{
Physical activity level in people with Down Syndrome: a \\ systematic review
}

\author{
El nivel de la actividad física en personas con Síndrome de \\ Down: una revisión sistemática
}

Artigo de Revisão

\section{RESUMO}

Objetivo: Investigar os níveis de atividade física em indivíduos com Síndrome de Down por meio de uma revisão sistemática. Métodos: A busca foi realizada nas bases de dados MEDLINE/PubMed, LILACS e SciELO, seguindo os critérios de elegibilidade e exclusão adotados. Resultados: Cinco estudos foram selecionados para a pesquisa, apresentando uma amostragem de 344 participantes de 3 a 70 anos de idade. Dentre os resultados encontrados, os estudos apresentaram uma variação dos níveis e prática de atividade física de acordo com cada faixa etária. De forma geral, os indivíduos com Síndrome de Down apresentam níveis particularmente baixos de atividade física e declínio acentuado com a idade. Conclusão: Os indivíduos com Síndrome de Down possuem baixo nível de atividade física, com índices inferiores às recomendações para uma vida ativa. Além disso, constatou-se que à medida que a idade avança, o nível de atividade física diminui. Verificou-se um baixo número de pesquisas com essa temática; dessa forma, recomenda-se que novos estudos sejam conduzidos, com o intuito de avaliar o nível de atividade física nesta população específica.

Descritores: Síndrome de Down; Deficiência Intelectual; Atividade Física.

\begin{abstract}
Objective: To investigate the physical activity levels in individuals with Down Syndrome, through a systematic review. Methods: The search was performed in MEDLINE/PubMed, LILACS, and SCiELO databases, following the adopted inclusion and exclusion criteria. Results: Five studies were selected for the study, with a sample of 344 participants aged 8-70 years. Among the findings, the studies presented varied physical activity practice and levels, according to each age group. In general, individuals with Down Syndrome present particularly low levels of physical activity and sharp decline with age. Conclusion: The individuals with Down Syndrome perform low level of physical activity, with lower rates than the recommendations for an active life. Moreover, it was found that, as age advances, the physical activity level decreases. There was a small number of researches addressing this issue. Thus, further studies should be conducted with the aim of assessing the level of physical activity in this population.
\end{abstract}

Descriptors: Down Syndrome; Intellectual Disability; Physical Activity.

1) Universidade do Estado de Santa Catarina - UDESC - Florianópolis (SC) -

Brasil
Recebido em: 28/08/2014 Revisado em: 28/10/2014 Aceito em: 17/12/2014 


\section{RESUMEN}

Objetivo: Investigar los niveles de la actividad fisica en individuos con Síndrome de Down a través de una revisión sistemática. Métodos: La búsqueda fue realizada en las bases de datos Medline/PubMed, Lilacs y Scielo según los criterios de elegibilidad y exclusión aprobados. Resultados: Cinco estudios fueron elegidos para la investigación con una muestra de 344 participantes entre 3 y 70 años de edad. De los resultados encontrados los estudios presentaron variación en los niveles y la práctica de actividad fisica según la franja de edad. En general, los individuos con el Síndrome de Down presentan niveles bajos de actividad física y disminución acentuada con la edad. Conclusión: Los individuos con el Síndrome de Down tienen bajo nivel de actividad fisica con indices abajo de las recomendaciones para una vida activa. Además, se constató que al paso que la edad avanza disminuye el nivel de actividad física. Se verificó un bajo número de investigaciones sobre este tema; por lo tanto, recomendase nuevos estudios con el objetivo de evaluar el nivel de actividad física de esta población especifica.

Descriptores: Síndrome de Down; Discapacidad Intelectual; Actividad Motora.

\section{INTRODUÇÃO}

A Síndrome de Down, ou trissomia do cromossoma 21 , é uma alteração genética causada por um erro na divisão celular durante a fase embrionária. Pessoas com essa síndrome, em vez de dois cromossomos no par 21, possuem três, resultando em sobre-expressão do gene. Trata-se de uma anomalia congênita e se constitui na causa genética mais comum de deficiência intelectual ${ }^{(1-3)}$.

O estado de saúde de adultos com deficiência intelectual é preocupante, com altos níveis de morbidade e mortalidade ${ }^{(4)}$. No caso de indivíduos com Síndrome de Down, os riscos de desenvolverem problemas relacionados à saúde são maiores quando comparados a outros grupos com deficiência intelectual ${ }^{(5)}$, tornando-se um importante grupo que necessita de investigação relacionada à saúde ${ }^{(6)}$.

As pessoas com Síndrome de Down têm tendência maior de apresentarem distúrbios respiratórios, osteoporose, problemas musculoesqueléticos, alguns tipos de câncer, doenças cardiovasculares, diabetes, obesidade e sedentarismo, contribuindo de forma independente para o desenvolvimento dessas doenças ${ }^{(7-9)}$. Destaca-se que a obesidade é bastante evidente, pois a prevalência de pessoas com sobrepeso ou obesas nesta população é 32\% maior que em indivíduos sem deficiência. Essa prevalência também é identificada na população de deficientes intelectuais, apontando valores que podem chegar a 59\% de obesos ${ }^{(10,11)}$.
O efeito protetor da atividade física no processo de adoecimento é bem estabelecido na literatura ${ }^{(12,13)}$ e inclui melhora da saúde geral, prevenção de doenças crônicas, aumento da autoestima e promoção da interação social ${ }^{(14,15)}$. Nesse contexto, mesmo que alguns problemas de saúde sejam mais evidentes nas pessoas com Síndrome de Down e deficiência intelectual, acredita-se que muitos poderiam ser prevenidos por meio da educação e promoção para um estilo de vida saudável ${ }^{(14,6)}$. Por esses motivos, existe a necessidade de se estabelecerem padrões específicos de atividades físicas para as pessoas com Síndrome de Down e deficiência intelectual, pelas recompensas dos benefícios ofertados ao bem-estar físico e mental na melhora da qualidade de vida desta população.

Atrelado a esse contexto, um estudo ${ }^{(16)}$ evidenciou que a participação em atividade física ou programas de treinamento com exercícios esteve relacionada a melhoras significativas no sistema cardiovascular, força muscular, equilíbrio, redução de resistência à insulina e obesidade abdominal em pessoas com Síndrome de Down. Entretanto, cabe destacar que a escassez desses programas, restrições nas instalações esportivas, dificuldades no transporte e acessibilidade têm sido citadas como barreiras para a prática de atividade física ${ }^{(16)}$. Ressalta-se que neste grupo específico ocorre a perda da capacidade funcional, por conta de uma baixa condição física, prejudicando o desempenho de uma variedade de tarefas cotidianas, incluindo o desempenho no trabalho ${ }^{(17,18)}$

Estudos apontam que a literatura é limitada e existe uma carência de pesquisas que abordem os temas de atividade física, pessoas com Síndrome de Down e outras deficiências $^{(19,20)}$. Tendo em vista os benefícios de um estilo de vida ativo nesta população, o presente estudo teve como objetivo investigar os níveis de atividade física em indivíduos com Síndrome de Down por meio de uma revisão sistemática.

\section{MÉTODOS}

A revisão sistemática de literatura foi realizada com recorte do período de 1969 a 2013. Definiu-se esse espaço de tempo devido à escassez de estudos encontrados num menor período de tempo previamente estipulado. Dessa forma, optou-se por realizar a busca em todos os artigos encontrados nas bases de dados utilizadas. Consultaramse as seguintes bases de dados virtuais: Medline/PubMed (Medical Literature Analysis and Retrieval System), Scielo (Scientific Electronic Library Online) e Lilacs (Literatura Latino-Americana e do Caribe em Ciências da Saúde), aplicando-se os mesmos descritores.

Utilizou-se o dicionário MeSH (Medical Subject Heading Terms) para definição dos descritores em inglês 
para pesquisa. Down Syndrome (MeSH), Mongolism (Entry Term) e Trisomy 21 (Entry Term) foram escolhidos para combinação com os termos Motor Activity (MeSH), Physical Activity (Entry Term), Locomotor Activity (Entry Term) e Sedentary Lifestyle (MeSH), resultando em um total de doze combinações de dois termos, utilizando-se o operador "and" para combinar os descritores. Em português, foi utilizado o vocabulário estruturado DeCS (Descritores em Ciências da Saúde) para aumentar o escopo da busca, acrescidos os descritores Trissomia do Cromossomo 21, Sedentarismo e Saúde da Síndrome de Down, além dos seus correspondentes em inglês. Utilizou-se o operador "e" para associação dos descritores, resultando em 24 combinações dos dois termos.

O processo de busca nas bases de dados eletrônicas, seleção dos estudos, leitura dos artigos e compilação das informações foi realizado pela pesquisadora principal do estudo. Replicou-se por um segundo pesquisador todo esse procedimento, de maneira cega e independente, não sendo identificadas divergências quanto à seleção final dos artigos.

Adotaram-se como critérios de elegibilidade para essa revisão: (1) a amostra deveria ser com indivíduos diagnosticados com Síndrome de Down, com ou sem deficiência intelectual; (2) ter como um dos objetivos do estudo mesurar o nível de atividade física; e (3) ser artigo original de pesquisa desenvolvida com seres humanos. Optou-se por não incluir artigos de revisão, monografias, dissertações, teses, resumos, capítulos ou livros e ponto de vista/opinião de especialistas, considerando a dificuldade de uma busca sistemática destes.

Com base nos critérios de busca e seleção, foram identificados 657 artigos. Destes, 592 foram excluídos com base na análise do título. Foram selecionados para leitura dos resumos 65 estudos; e para leitura na íntegra, 29. Após a leitura dos artigos na íntegra, cinco trabalhos atenderam aos critérios de inclusão que contemplaram esta revisão. Todo o processo de seleção dos estudos está descrito na Figura 1.

\section{RESULTADOS}

Identificaram-se inicialmente 657 estudos, dos quais foram selecionados 29 para leitura do texto na íntegra; destes, apenas cinco atenderam aos critérios de elegibilidade. Dos artigos excluídos, destacam-se os seguintes motivos: não apresentarem intervenções com exercícios físicos; serem estudos de revisão sistemática ou de literatura; referirem-se apenas a indivíduos com deficiência intelectual ou autismo; pesquisarem apenas as barreiras à prática da atividade física; abordarem força e desempenho em tarefas cotidianas; ou não abordarem a atividade física em específico, e sim aspectos de condições de saúde.
Dentre os estudos incluídos, o método mais utilizado para avaliar a prática de atividade física foi o acelerômetro, seguido pelo pedômetro e questionário. Os estudos incluíram crianças, adolescentes, adultos e idosos com Síndrome de Down, independentemente do sexo, e um estudo com diagnóstico de Síndrome de Down com deficiência intelectual. Entre os estudos, dois foram realizados no Brasil, dois nos Estados Unidos da América e um na Inglaterra.

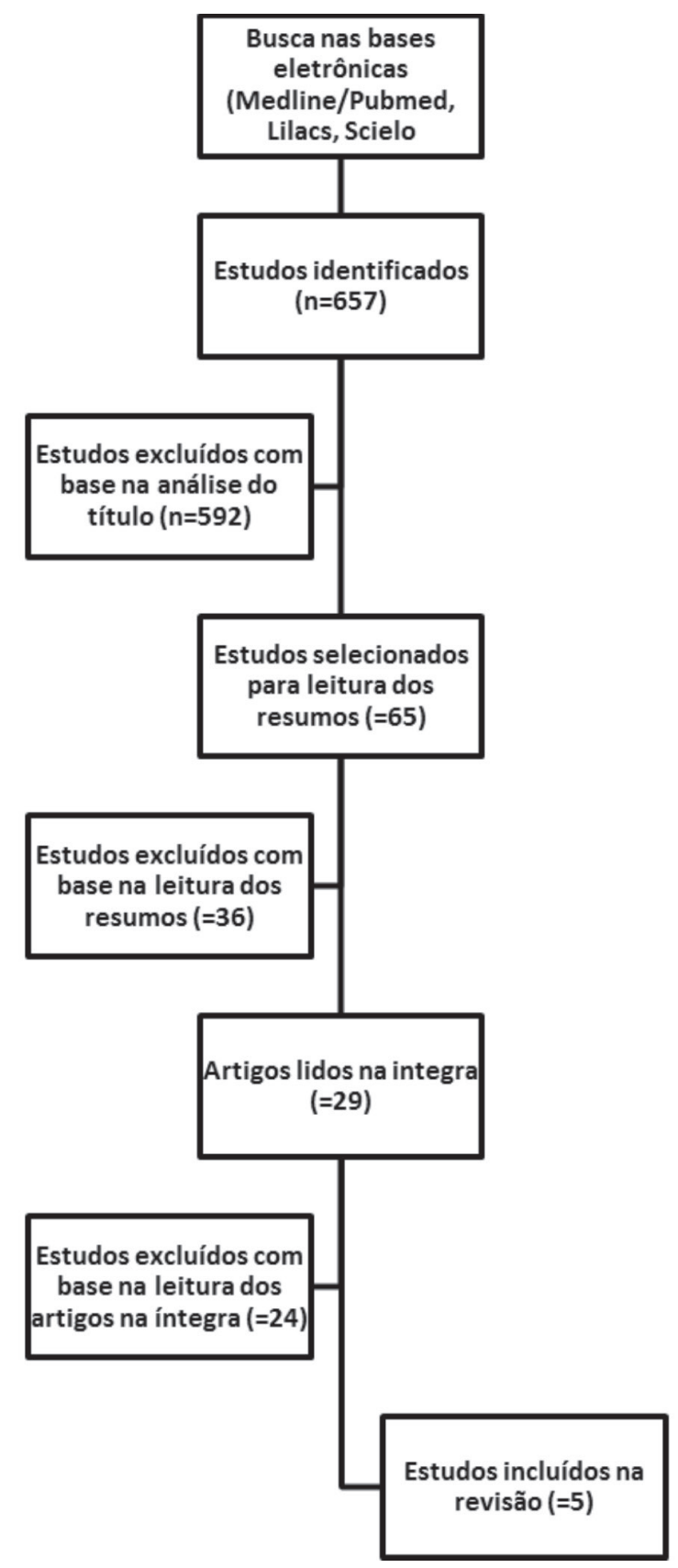

Figura 1 - Processo de seleção e revisão dos estudos. Florianópolis-SC, 2014. 


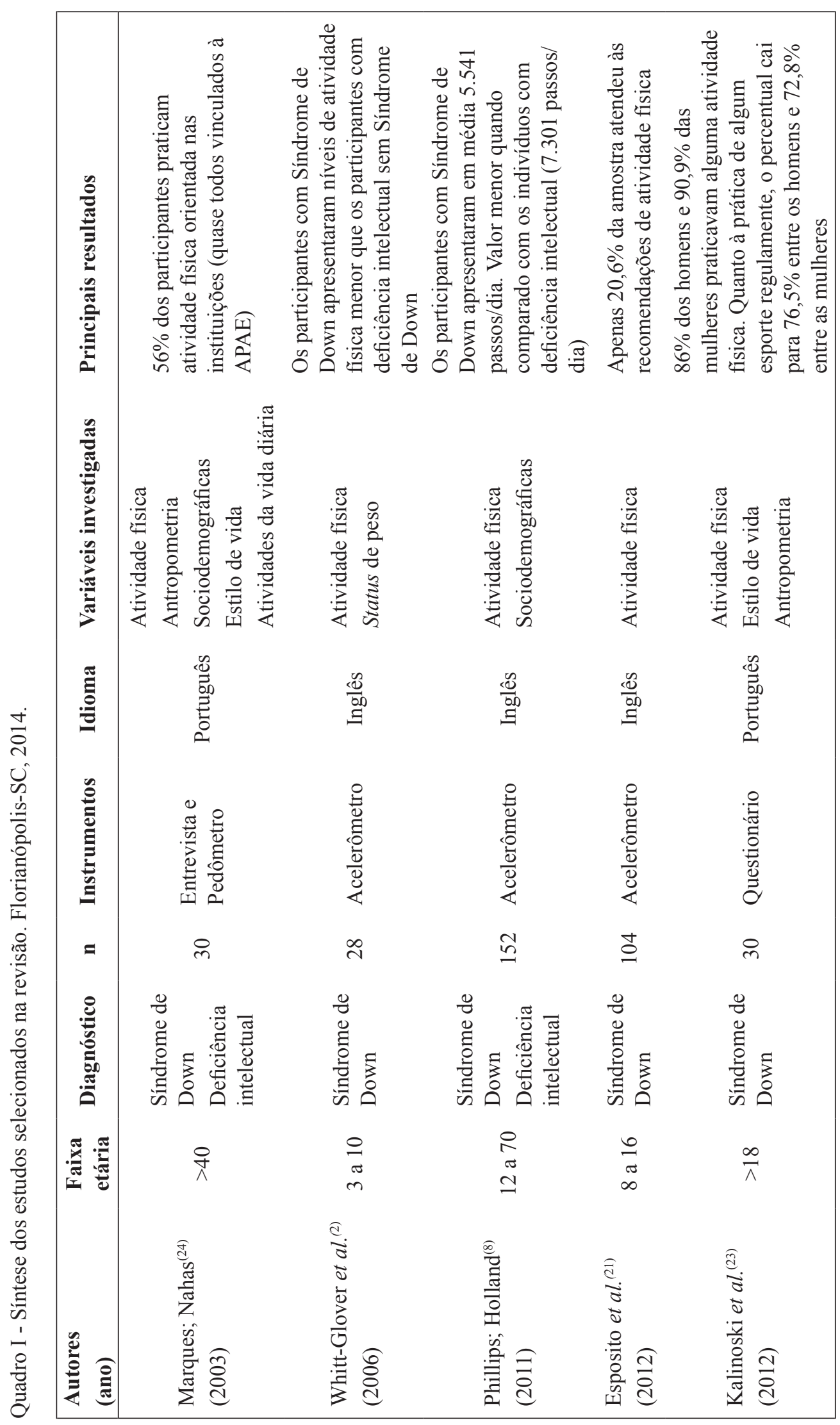


Em dois estudos ${ }^{(8,21)}$, os autores afirmaram que os níveis de atividade física, de acordo com a faixa etária da amostra pesquisada, estão abaixo dos recomendados pela Organização Mundial de Saúde (OMS) ${ }^{(22)}$. Já em outros dois estudos foram identificados valores satisfatórios de atividade física ${ }^{(2,23)}$.

Relacionou-se a boa frequência de participação em atividade física com as realizadas em instituições especiais, quase todas vinculadas à Associação de Pais e Amigos dos Excepcionais (APAE), por meio das aulas de Educação Física. Porém, quando o nível de atividade física foi avaliado no momentos de lazer, classificou-se a amostra estudada com baixo nível de atividade física ${ }^{(24)}$. Outras características dos estudos selecionados estão descritas no Quadro I.

\section{DISCUSSÃO}

Os principais achados indicaram que, de forma geral, indivíduos com Síndrome de Down possuem um baixo nível de atividade física. Sabe-se que uma vida ativa é benéfica para todas as pessoas, incluindo essa população. Os benefícios incluem melhora na saúde, prevenção de doenças crônicas, aumento da autoestima e a promoção da interação social ${ }^{(14-16,25)}$.

Identificaram-se nesta revisão dois $\operatorname{estudos}^{(2,23)} \mathrm{em}$ que a participação em atividade física foi satisfatória. No primeiro $^{(2)}$, foi comparado o nível de atividade física em crianças com Síndrome de Down e seus irmãos sem esse distúrbio genético. As crianças com Síndrome de Down eram mais novas e tinham o índice de massa corporal (IMC) mais elevado do que seus irmãos. Também se constatou que o nível de atividade física de intensidade leve e moderada em ambos os grupos era adequado, porém, as crianças com Síndrome de Down participavam de menos atividades de intensidade vigorosa. $\mathrm{O}$ estudo concluiu que a participação em atividades de intensidade vigorosa na infância pode ser apropriada para a prevenção da obesidade e promoção de saúde ao longo da vida.

No segundo estudo ${ }^{(23)}$, o padrão de atividade física na população estudada apresentou valores satisfatórios $(81,6 \%$ dos homens e $90,9 \%$ das mulheres praticavam alguma atividade física). Em outro estudo ${ }^{(24)}$, verificou-se que 56,3\% dos sujeitos faziam atividade física orientada na instituição e 26,6\% não realizavam nenhum tipo de atividade física. No entanto, ambos os estudos ${ }^{(23,24)}$ apresentaram como justificativa e limitação o fato de a amostra estudada participar de projetos de extensão e estar matriculada na escola, o que aumenta as oportunidades para a prática de atividade física.

Vale ressaltar que uma pesquisa ${ }^{(24)}$, ao analisar o nível de atividade física diretamente, registrando a quantidade de passos utilizando um pedômetro, observou uma média total de 4.018 passos/dia, sendo os indivíduos classificados na categoria menos ativos (menos de 5.000 passos/dia). A baixa participação em atividades físicas regulares é uma preocupação nesta população. Ser fisicamente ativo pode ter uma série de benefícios para a saúde, e a maioria das atividades também traz benefícios sociais, como encontro com amigos e diversão ${ }^{(26,30)}$.

Em estudo publicado em 2012(21), indivíduos com Síndrome de Down avaliados passaram a maioria dos dias em atividades sedentárias e apenas $20,6 \%$ da amostra atendeu às recomendações de atividade física. Outro estudo, desenvolvido em $2011^{(8)}$, também verificou que os indivíduos não atendiam às recomendações para a saúde. Para a $\mathrm{OMS}^{(22)}$, crianças e jovens devem acumular pelo menos 60 minutos de atividade física moderada e vigorosa em todos os dias da semana. Para os adultos de 18 a 64 anos, a recomendação é o acúmulo de pelo menos 150 minutos de atividade física de intensidade moderada ou 75 minutos de atividade física vigorosa durante a semana.

Esses resultados estão de acordo com a literatura que indica baixos níveis de aptidão física em indivíduos com Síndrome de Down, alta prevalência de obesidade em crianças, adolescentes e adultos, o que pode estar relacionado ao estilo de vida sedentário ${ }^{(26-30)}$.

Com relação aos padrões de atividade física, três estudos observaram uma diminuição no nível de atividade física com o aumento da idade, sugerindo que quanto maior a idade menor o nível de atividade física ${ }^{(8,21,24)}$. Uma possível explicação para essa redução pode estar relacionada às atividades intermitentes durante a infância. À medida que as crianças aumenta sua idade, essas atividades informais diminuem e são substituídas por atividades mais estruturadas $^{(21)}$. Para indivíduos com Síndrome de Down, a falta de atividades estruturadas tem sido uma razão para a não participação em atividade física ${ }^{(16)}$.

Entre os artigos incluídos neste estudo, a maioria deles utilizou medidas diretas para estabelecer o nível de atividade física. Nenhuma pesquisa com delineamento longitudinal foi selecionada. Sabe-se da dificuldade em realizar pesquisas com essa metodologia, especialmente em populações especiais.

Esses dados se consolidam pela falta de incentivos dos pais a compreender os benefícios e a manutenção desses indivíduos nos programas de atividade física. Por conseguinte, sabe-se que as atitudes dos familiares influenciam o comportamento e desenvolvimento do estilo de vida dos seus filhos.

Como forma de aumentar a participação em atividades mais ativas, sugere-se o desenvolvimento de políticas públicas que promovam a saúde e o bem-estar dessa população em todas as faixas etárias. Profissionais da área 
da saúde, educadores, familiares e indivíduos que trabalham diretamente com essa população são fundamentais nesse processo e devem incentivar ao máximo a participação em programas de atividade física.

\section{CONCLUSÃO}

Os indivíduos com Síndrome de Down possuem baixo nível de atividade física, com índices inferiores às recomendações para uma vida ativa. Além disso, constatouse que à medida que a idade avança, o nível de atividade física diminui. Verificou-se um baixo número de pesquisa com essa temática e, dessa forma, recomenda-se que novos estudos sejam conduzidos, com o intuito de avaliar o nível de atividade física nesta população específica.

\section{REFERÊNCIAS}

1. Rozzen NJ, Patterson D. Down's Syndrome. Lancet. 2003;361(9365):1281-9.

2. Whitt-Glover MC, O’Neill KL, Nicolas S. Physical activity patterns in children with and without Down Syndrome. Pediatr Rehabil. 2006;9(2):158-64.

3. Mahy J, Shields N, Taylor NF, Dodd KJ. Identifying facilitators and barriers to physical activity with Down Syndrome. J Intellect Disabil Res. 2010;54(9):795805.

4. Bittles AH, Petterson BA, Sullivan SG, Hussain R, Glasson EJ, Montgomery PD. The influence of intellectual disability on life expectancy. J Gerontol A Biol Sci Med Sci. 2002;57(7):470-2.

5. Pueschel SM, Pueschel JK, editors. Biomedical concerns in persons with Down Syndrome. Baltimore: Paul H. Brookes; 1992.

6. Saad SN. Preparando o caminho da inclusão: dissolvendo mitos e preconceitos em relação à pessoa com Síndrome de Down. Rev Bras Ed Esp. 2003;9(1):57-78.

7. Prasher VP, Janichi MP. Future prospects: a challenge to promote wellness. In: Prasher VP, Janicki MP. Physical health of adults with intellectual disabilities. Oxford: Blackwell; 2002.

8. Phillips AC, Holland AJ. Assessment of objectively measured physical activity levels in individuals with intellectual disabilities with and without Down's Syndorme. PLos ONE. 2011;6(12):e28618.

9. Hamilton MT, Hamilton DG, Zderic TW. Role of low energy expenditure and sitting in obesity, metabolic syndrome, type 2 diabetes, and cardiovascular disease. Diabetes. 2007;56(11):2655-67.

10. Rimmer JH, Braddock D, Fujijura G. Prevalence of obesity in adults with mental retardation: Implications for health promotion and disease prevention. Ment Retard. 1993;31(2):105-10.

11. Illingworth K, Moore KA, McgillivrayJ. The development of the Nutritional and Activity Knowledge Scale for use with people with an intellectual disability. J Appl Res Intellect Disabil. 2003;16(2):159-66.

12. Dunn AL, Trivedi MH, O’Neal H. A. Physical activity dose-response effects on outcomes of depression and anxiety. Med Sci Sports Exerc. 2001;33 (6 Suppl):S587-97.

13. National Preventative Health Taskforce (AUS). Australia: The healthiest country by 2020. National preventative health strategy - The roadmap for action. Canberra: Australian Governmen; 2009.

14. Jobling A. Life be in it: lifestyle choices for active leisure. Downs Syndr Res Pract. 2001;6(3):117-22.

15. Rimmer JH, Riley B, Wang E, Rauwrth A, Jurkowski J. Physical activity participation among persons with disabilities: barriers and facilitators. Am J Prev Med. 2004;26(5):419-25.

16. Menear K. Parents' perceptions of health and physical activity needs of children with Down Syndrome. Downs Syndr Res Pract. 2007;12(1):60-8.

17. Carmeli E, Kessel S, Bar-Chad S, Merrick J. A comparison between older persons with Down Syndrome and a control group: clinical characteristics, functional status and sensorimotor function. Downs Syndr Res Pract. 2004;9(1):17-24.

18. Cowley PM, Ploutz-Snyder LL, Baynard T, Hefferman $\mathrm{K}$, Jae SY, Hsu S, et al. Physical fitness predicts functional tasks in individuals with Down Syndrome. Med Sci Sports Exerc. 2010;42(2):388-93.

19. Fernhall B, Unnithan VB. Physical activity, metabolic issues, and assessment. Phys Med Rehabil Clin N Am. 2002;13(4):925-47.

20. Foley JT, Bryan RR, Mccubbin JA. Daily physical activity levels of elementary school-aged children with and without mental retardation. J Dev Phys Disabil. 2008;20(4):365-78.

21. Esposito PE, Macdonald M, Hornyk EJ, Ulrich D. A. Physical activity patterns of youth with Down Syndrome. Intellect Dev Disabil. 2012;50(2):109-19. 
22. World Health Organization et al. Global recommendations on physical activity for health. 2010.

23. Kalinoski AX, Marques AC, Jung LG, Xavier GB. Prevalência de fatores de risco da síndrome metabólica em adultos com síndrome de Down na cidade de Pelotas. Rev Bras Ativ Fís Saúde. 2012;17(5):396-402.

24. Marques AC, Nahas MV. Qualidade de vida de pessoas portadoras de Síndrome de Down, com mais de 40 anos, no Estado de Santa Catarina. Rev Bras Ciênc Mov. 2003;11(2):55-61.

25. Balic MG, Mateos EC, Blasco CG, Fernhall B. Physical fitness levels of physically active and sedentary adults with Down syndrome. Adap Phys Act Quarterly. 2000;17(3):310-21.

26. Buckley S. Increasing opportunities for physical activity. Downs Syndr Res Pract. 2007;12(1):18-9.

27. Fernhall B, Pitetti KH, Rimmer JH, Mccubbin JA, Rintala P, Millar L. Cardiorespiratory capacity of individuals with retardation including Down Syndrome. Med Sci Sports Exerc. 1996;28(3):366-71.
28. Rubin S, Rimmer JH, Chicoine B, Braddock D, Mcguire D. Overweight prevalence in persons with Down Syndrome. Ment Retard. 1998;36(3):175-81.

29. Varela AM, Sardinha LB, Pitetti KH. Effects of an aerobic rowing training regimen in young adults with Down Syndrome. Am J Ment Retard. 2001;106(2):13544.

30. Melville CA, Cooper SA, Mcgrother CW, Thopr CF, Collacott R. Obesity in adults with Down Syndrome: a case control study. J Intellect Disabil Res. 2005;49(Pt 2):125-33.

\section{Endereço para correspondência:}

Ana Paula Pietro Nobre Montoro

Centro de Ciências da Saúde e do Esporte - CEFID

Universidade do Estado de Santa Catarina - UDESC

Rua Paschoal Simoni, 358

Bairro: Coqueiros

CEP: 88080-350 - Florianópolis - SC - Brasil

E-mail: anappnmontoro@hotmail.com 